\title{
La diversidad cultural y las políticas culturales: la distancia entre la intolerancia y la integración
}

Dra. Delia Fajardo Salinas*

\section{Resumen}

En este artículo perfilamos la evolución histórica de varias categorías que definen paradigmas sobre las distintas maneras de concebir y tratar con la diversidad cultural en la sociedad, enfocando cómo en el ejercicio de definir dichos paradigmas subyace precisamente una lucha entre grupos o clases sociales políticamente confrontados. La revisión de esta lucha simbólica en la dimensión teórica finaliza con la categoría "intertransculturalidad", valorada como una propuesta muy atractiva para fundamentar el diseño de políticas educativas orientadas a legitimar y potenciar el desarrollo de la educación intercultural bilingüe.

Palabras Clave: pluralismo cultural, multiculturalismo, interculturalidad, educación, intertransculturalidad.

\begin{abstract}
In this article we outline the historical evolution of various categories that define paradigms about the different ways of thinking and dealing with cultural diversity in society, focusing on how the exercise of underlying paradigms precisely define such a struggle between groups and / or classes social and politically confronted. The review of this symbolic struggle in the theoretical dimension ends with the category "intertransculturalidad" valued as a very attractive proposition to support the design of educational policies aimed at legitimizing and promoting the development of intercultural bilingual education.
\end{abstract}

\footnotetext{
* Docente, Departamento de Letras y Lenguas, Universidad Pedagógica Nacional Francisco Morazán, demafasa@yahoo.com
} 
Key words: cultural pluralism, multiculturalism, interculturalism, intercultural education, inter transculturality.

\section{La Diversidad: Un fenomeno con varias miradas}

La diversidad es un fenómeno inherente a la evolución de la especie humana, pero la forma en que la propia especie se ha enfrentado a ello ha tomado igualmente diversidad de caminos a lo largo de la historia. El racismo, la xenofobia, el asimilacionismo, la fusión cultural, el pluralismo cultural, el multiculturalismo y, el más reciente, la interculturalidad son esos caminos coyunturales que han canalizado, o intentan hacerlo, las reacciones de los seres humanos ante la Otredad; distintas posiciones sobre cómo resolver las relaciones entre grupos sociales diferentes, ya sea por pertenencia a una étnia, lengua, sexualidad, cultura, religión o pertenencia a una nación.

Cada posicionamiento tiene distintas consecuencias. Así, los dos primero son modelos de exclusión social basados en la idea de la superioridad racial, que conlleva la discriminación y la segregación, y por ello han sido el origen de los eventos más dramáticos que han marcado la modernidad: el holocausto, el apartheid, la limpieza étnica.

El asimilacionismo y la fusión cultural suponen una superación de esas posturas tan extremistas, pero su propuesta de inclusión afecta siempre negativamente a una de las partes, o ambas, porque implican suplantación y pérdida de la cultura original que se trae al encuentro social, bajo un sentido siempre de infravaloración. Conocido en inglés como 'melting pot', la fusión cultural fue objeto de profundas críticas por parte de la antropología latinoamericana, que en su lugar propuso el término 'transculturación' (Ortiz, 1963; Rama, 1987), para rescatar de la marginación simbólica los valores que la cultura latinoamericana aportaba en esa fusión.

El pluralismo cultural y el multiculturalismo avanzan un escalón más cercano hacia el ideal democrático; pero estos modelos todavía ocultan en su gentil política otra forma de manipulación ideológica en el juego de las relaciones desiguales de poder, como se puede detectar al revisar sus orígenes en la información que aportan 
Malgesini y Giménez (2000). Estos autores señalan que el pluralismo cultural fue una iniciativa europea posterior a la II Guerra Mundial, para orientar los procesos de reconstrucción en los países europeos que gozaban de estabilidad democrática y en Estados Unidos. Según los autores, en este momento histórico, fue evidente: 1) el fracaso de los modelos previos: asimilacionismo y fusión cultural; 2) el reconocimiento de la persistencia étnica, es decir, de grupos étnicos diferentes a pesar de las predicciones que suponían los modelos de suplantación cultural; y 3) la afluencia migratoria a esos países.

Definen pluralismo cultural como la "ideología o modelo de organización social que afirma la posibilidad de convivir armoniosamente en sociedades grupos o comunidades étnica, cultural, religiosa o lingüísticamente diferentes" (ibid.). Lo caracterizan por su valoración positiva de las diferencias, invitar a su conservación y suponer que la diversidad se recrea, sin llegar a la asimilación o a la fusión. Además, promueve la idea de que todos los grupos convergentes tienen algo que ofrecer y aprender de los otros, a través de su interacción.

Esta aceptación positiva de las diferencias ha evolucionado a la formulación de la diferencia como derecho, entendida ésta en la mayor amplitud del término en relación a: valores, religión, etnicidad, género, patria, etc. $Y$ en segundo lugar, el reconocimiento de la igualdad de derechos y deberes. Es en esta etapa que se utiliza más el término multiculturalismo para referirse a este conjunto de principios, como sinónimo de pluralismo cultural y es en la actualidad, a nuestro parecer, el que ha quedado más asentado en los discursos oficiales y de la sociedad civil. Por ejemplo, el multiculturalismo es utilizado por la ONU como su marco de referencia, y forma parte de la política de países como Canadá, Estados Unidos, Inglaterra, Holanda, Nueva Zelanda, Bolivia, entre otros.

Esta evolución nos hace aceptar la observación de Ruth Moya (2004), de Ecuador, de que el concepto pertenece a una iniciativa anglosajona, para "tratar de responder a las situaciones de adecuada inserción cultural en la compleja matriz sociocultural del entorno europeo o norteamericano" (17). Por ello, desde una perspectiva crítica, esa iniciativa se ha denunciado como una estrategia del proceder 
políticamente correcto pero sin una agenda explícita y concreta, que en realidad busca distribuir el poder entre los grupos étnicos hegemónicos, hasta lograr que la estructura política se acomode a estos grupos en el sistema democrático (Malgesini y Giménez, 2000: 326). En términos más directos, Briones (2002), de Argentina, se refiere al multiculturalismo como la política de Estado de países centrales nor-atlánticos "para contener reclamos de respeto a la diferencia" (1).

Como bien lo señala Díaz-Couder (1998), de México, dependiendo de la forma en que se entienda la multiculturalidad, así se define un proyecto de nación y educación específico. Por ello identifica dos tipos: un multiculturalismo de la tolerancia que hace un reconocimiento de la diferencia y concede el derecho 'privado' de conservar la lengua y cultura propia y supone un trato no discriminatorio; además, el Estado asume la responsabilidad de preservar esas culturas, definidas entonces como patrimonio nacional, como lo son los objetos históricos o artísticos; dicha preservación se traduce, por ejemplo, en apoyo a las tradiciones consideradas 'floklóricas' y en implementar programas educativos bilingües de transición que finalmente llevan a la asimilación de la lengua y cultura oficial dominante, es decir, "se acaba la tolerancia" (29).

El otro modelo multicultural sería realmente pluralista porque otorga una nueva condición política a los pueblos o comunidades minorizados debido a su diferencia lingüística, étnica y cultural, como sujetos de derecho 'público'. Esto significa que además de derechos culturales, poseen derechos políticos de interés nacional, colectivo y no individual, lo cual se traduce en una verdadera promoción y desarrollo de la lengua, cultura y existencia social de los pueblos implicados, en un plano de equidad.

En palabras de Duarte et al. (1999), del Proyecto Q'Anil en Guatemala, este tipo de multiculturalidad funciona con base en dos principios: el derecho a la ciudadanía (igualdad de derechos, deberes y oportunidades), y el derecho a la diferencia (respeto a las diferencias culturales). Es decir, la vida social y pública debe organizarse en función de garantizar beneficios sociales para toda 
la población: educación, salud, participación ciudadana y pública, asistencia jurídica, trabajo social, etc. Según los autores, para esos años de finales del siglo XX, ya era posible ver efectos de esto en Guatemala ya que el respeto a las culturas no hegemónicas ganaba mayor espacio, mediante acciones concretas: la reforma de la escuela, la lucha contra el racismo y la discriminación, y la adecuación del Estado a su entorno pluricultural y multilingüe.

Con la anterior referencia a los análisis de varios autores latinoamericanos, queremos evidenciar que, al pasar al contexto latinoamericano, el multiculturalismo ha sufrido resignificaciones debido a que se instala en el contexto del movimiento indígena y los procesos de cambio para pasar de un Estado monocultural a uno multicultural. Con el antecedente de la Declaración Universal de los Derechos Humanos en 1948 y el pluralismo cultural que conduce a entender la diversidad como una riqueza, un recurso positivo para el desarrollo en lugar de un problema, el movimiento indígena logra hacer de su identidad, fortalecida simbólicamente, un potente recurso: "El conjunto de características y peculiaridades indígenas fue promovido por los movimientos indígenas, en los ochenta y sobre todo en los noventa, como mecanismos de aglutinación y de organización política que les permitieran asumirse como colectivos sociohistóricos con su propia identidad, la misma que se convertía en un recurso de la lucha social" (Moya, 2004: 15-16).

Por este condicionamiento en los procesos políticos, similar resignificación ocurre con el concepto de interculturalidad, el cual surgió precisamente por el descontento ante las políticas multiculturales 'tolerantes' que no hacían sino posponer el cumplimiento de la añorada promesa de equidad social.

\section{La Interculturalidad}

Malgesini y Giménez (2000) consideran que la interculturalidad se introdujo a partir de reconocer un hecho incuestionable: la interacción entre culturas ha existido siempre, por lo que, si bien es cierto que se deben tomar en cuenta las diferencias, los puntos de convergencia resultan una plataforma más viable para lograr esa deseada unidad en la diversidad. Así, la interculturalidad cambió el 
peso en la balanza y orientó la atención hacia los vínculos que ya unen a distintos grupos culturales que confluyen en un mismo espacio territorial, los valores compartidos, las normas de convivencia ya legitimadas y aceptadas y los intereses comunes para el desarrollo local “...ya no en el plano de lo que es sino de lo que debe ser, para poder hacer una propuesta de sociedad pluricultural en el sentido de la convivencia de todas las formas de vida” (253).

Tanto en los países europeos, norteamericanos y latinoamericanos, el término interculturalidad surgió del campo educativo y, según lo describen estos autores, éste se expandió paulatinamente a otros campos (procesos de comunicación, modelos de integración y convivencia social, etc.). Las primeras formulaciones sobre interculturalidad en el campo educativo hablan de una propuesta de actuación, no de un fenómeno objetivable o un hecho aislado, lo cual resulta obvio porque el aula es un espacio de intensa interacción más que de estudios teóricos, es decir, es el sitio exacto donde se hace evidente que las ideas del pluralismo no conducían a solucionar la problemática producto de esta situación de contacto inevitable, y que no se resolvía con la buena intención integradora, sin estrategias claras que atendieran conflictos puntuales.

Malgesini y Giménez resaltan que se vio:

"la necesidad de renovar radicalmente los currículos monoculturales, de no separar los grupos en la escuela, de no presentar como monolíticas las culturas, de llevar al terreno educativo el enriquecimiento que supone la presencia de bagajes culturales diferenciados, y, en definitiva, de intervenir educativamente sobre la interacción en la escuela y de preparar para la interacción en la sociedad" (254).

Esta es una visión de la propuesta intercultural en el contexto de países receptores de inmigrantes. Para referirnos a los planteamientos en el contexto latinoamericano, podemos retomar un concepto básico que define la interculturalidad como una categoría que "remite a la diversidad étnica, a los particularismos culturales y a las formas en que estas relaciones actúan en la convivencia social" (Moya, 2004: 20). Este concepto se puede enriquecer mucho más al considerar distintas dimensiones de aplicabilidad del término, y nos interesa destacar sobre todo la dimensión sociopolítica del 
concepto, ya que nos parece su aspecto central, en relación a todos los demás sentidos que adquiere -especialmente en la educación. Dichos sentidos dependen estrechamente de los actores sociales, es decir, desde la posición sociopolítica desde donde se enuncie un concepto de interculturalidad, como bien advierte Moya:

“...la de interculturalidad es una noción compleja que puede entenderse con propiedad a partir del análisis del contexto en el cual este concepto se debate y aplica [y debido a ello] el ejercicio de la interculturalidad tiene características marcadas por la historia y la idiosincrasia de los pueblos involucrados" (ibid.).

A continuación presentamos una recopilación de varias definiciones. Comenzaremos con la Zimmermann (1997), quien considera que la interculturalidad es:

\begin{abstract}
"un conjunto de actividades y disposiciones destinadas a terminar con los aspectos y resultados negativos de las relaciones interculturales conflictivas. También se puede entender como una contribución al establecimiento de relaciones pacíficas, al mutuo entendimiento, al derecho a vivir la propia cultura, a la tolerancia, y, en fin, a la autodeterminación cultural" (117).

En palabras de López (1997), la interculturalidad como política educativa es:

"una alternativa al enfoque homogeneizante del sistema educativo tradicional occidental. Como tal, busca: una transformación de las relaciones sociales ante la diversidad desde la equidad, pertinencia y relevancia curricular; ser una herramienta para la construcción de una ciudadanía inclusiva en lugar de excluyente" (57).
\end{abstract}

Los mensajes de crítica social son evidentes en ambas definiciones y demuestran la interiorización del principio de igualdad de todos los seres humanos, por parte del movimiento indígena. Moya explica que sobre esta plataforma se pudo denunciar la injusticia e inmoralidad de las condiciones de violencia social y física de la que han sido víctima (racismo, marginación, exclusión, represión, etc.) y perfilar un imaginario indígena sobre la posibilidad de diálogo sin jerarquías, de igual a igual. Pero el agravante es que este imaginario ha chocado siempre con "la sordera endémica de las etnocracias" (2004: 12), que han sabido mantener las condiciones de gobernabilidad a favor de sus intereses. Esto conlleva un desencanto 
que luego ha conducido a nuevas estrategias de negociación; por ejemplo, en el contexto específico de los Acuerdos de Paz en Guatemala (1996), el movimiento indígena reclamó que se observara una verdadera coherencia con la interculturalidad, definida explícitamente como 'poder compartido' (ibid.).

Desde la dimensión propiamente pedagógica, López define la interculturalidad según dos usos instrumentales: para crear una nueva pedagogía de la diversidad, y como metodología para una revisión y renovación del currículo escolar, a partir de las relaciones entre conocimiento, cultura y lengua (ibid.: 58 ).

Podemos añadir una dimensión pragmática, es decir, el sentido que hace de la interculturalidad un proceso inmediato, un plan de acción, en lugar de una meta lejana. Este nos parece un mejor posicionamiento para enfrentar los siguientes metas de aprendizajes a lograr desde la escuela, identificados por Duarte et al. (1999):

- El reconocimiento del Otro como interlocutor en igualdad

- Conocer las otras culturas distintas a la propia

- Valorar la diversidad cultural en los lindes de la nación

- Superar la desconfianza, temores, prejuicios, estereotipos

- Aspirar a un aprendizaje mutuo, con la voluntad para hacerlo

- Procurar una comunicación efectiva

- Optar por el trabajo conjunto y efectivo en la organización de la vida social, en función sobre todo, de perfilar un proyecto válido y consensuado de reconfiguración del Estado-nación.

Dicho en otras palabras, estos comportamientos marcarían la pauta de relaciones interculturales, caracterizadas por el intercambio armónico, la convivencia pacífica, el interés positivo por el Otro, la comunicación efectiva y el aprendizaje mutuo, la confianza, la cooperación, la regulación pacífica del conflicto mediante el diálogo; es decir, atender tanto características de las relaciones humanas como valores y actitudes de los sujetos involucrados (ibid.: 25).

La validez de la interculturalidad como paradigma social para alcanzar estos objetivos se justifica con varios argumentos que Zimmermann (1997) explica sólidamente: no es solamente una consecuencia lógica 
de los derechos humanos, como ya se vio, sino que también de la ética porque quien no respeta el derecho ajeno, no puedo reclamar el respeto del propio; entonces si las personas que confluyen en un conglomerado social vienen de distintas trayectorias históricas y perfiles culturales muy diferentes, y lo hacen insertos en relaciones de poder desiguales, se debe empezar por cambiar estas condiciones.

Desde una perspectiva económica, este autor argumenta que los efectos colaterales de los otros paradigmas para confrontar la diversidad (opresión cultural, escolarización deficiente, asimilación conflictiva, deserción, baja escolaridad), lo que generan es personas lesionadas en su identidad que no ofrecen la misma potencialidad para unirse a las fuerzas productivas de su país, porque "una persona con identidad deformada se ocupa inconscientemente de sus problemas de identidad" (1997: 118). Es decir, el bienestar económico de la nación depende directamente del bienestar psicológico (entiéndase identitario) de cada uno de sus miembros.

Por tanto, un tercer argumento postula que es preferible aspirar a la construcción de una identidad colectiva saludable que redunda positivamente en la autoestima individual y en reducir "la posibilidad de futuros conflictos, que son malos para la vida social en general y para la economía en particular” (ibid.: 118).

Finalmente, como ejemplo de lo que sería una definición de interculturalidad en un documento oficial educativo, nos parece conveniente mostrar la propuesta de Duarte et al. (1999), del Proyecto Q'Anil, en Guatemala, para quienes la interculturalidad es:

"Un planteamiento pluralista sobre las relaciones humanas que debería haber entre actores culturalmente diferenciados en el contexto del Estado democrático y participativo y de la Nación pluricultural, multilingüe y multiétnica; la promoción sistemática y gradual, desde el Estado y desde la sociedad civil, de espacios y procesos de interacción positiva que vayan abriendo y generalizando relaciones de confianza, reconocimiento mutuo, comunicación efectiva, diálogo y debate, aprendizaje e intercambio, regulación pacífica del conflicto, cooperación y convivencia, sobre la base de tres principios: 
1. el principio de ciudadanía, que implica el reconocimiento pleno y la búsqueda constante de igualdad real y efectiva de derechos; responsabilidades, oportunidades, así como la lucha permanente contra el racismo y la discriminación;

2. el principio del derecho a la diferencia, que conlleva el respeto a la identidad y derechos de cada uno de los pueblos, grupos étnicos y expresiones socioculturales de Guatemala; y

3. el principio de unidad en la diversidad; concretado en la unidad nacional, no impuesta sino construida por todos y asumida voluntariamente" (26-27).

\section{De camino hacia la transculturación}

No está de más insistir en que todo este edificio teórico para justificar la interculturalidad como modelo idóneo para la organización de la convivencia social y fundamento para el diseño de políticas educativas, tiene como basamento original el concepto antropológico de cultura, y que dos aspectos derivados de dicho concepto cobran especial relevancia en esta discusión: la existencia de heterogeneidad al interior de una misma cultura, y su carácter cambiante. Incluso, si partimos de esta "visión dinámica de la cultura y en el entendimiento que en el mundo de hoy y en la interdependencia que lo caracteriza, no es posible diseñar o establecer límites o linderos absolutos entre culturas diferentes" (López, 1997: 58).

Desde el contexto europeo, A. Perotti (1994) ya había propuesto una definición de sociedad intercultural en tanto proyecto político orientado a desarrollar una nueva síntesis cultural. Esto implica una "generación intencionada, planificada o inducida, de algo nuevo, de expresiones culturales nuevas", pero sin repetir el modelo de fusión cultural, ya que Perotti aclara que su propuesta "supone la elaboración de modelos originales procedentes de las culturas en presencia -sin por ello dejar reducir a ninguno de ellos-que se incorporan a la cultura nacional de base reforzada y renovada" (66).

Ampliando sus ideas, Perotti añade que "esos modelos originales elaborados en el proyecto intercultural a partir de las culturas en presencia-se incorporan a 'la cultura nacional de base reforzada y 
renovada'. Estamos pues ante una propuesta que asume la existencia de culturas nacionales y que busca la unidad social de la nación" (ibid). Vemos que para este autor, la interculturalidad como proyecto sociopolítico es considerada como una de las vías para reconfigurar el modelo tradicional del nacionalismo, apegado a ideales de homogeneidad, y abre el camino para pensar que la heterogeneidad tampoco puede permanecer pétrea. Gracias a esta formulación, a las características ya enumeradas de la interculturalidad podemos añadir la creación del ambiente propicio para la recreación de las culturas que confluyen en una sociedad, y con ello la emergencia de una nueva síntesis cultural.

Esta nueva síntesis ya había recibido una denominación en el trabajo antropológico del cubano Fernando Ortiz, cuando propuso en su momento el neologismo transculturación, a partir de la palabra transmutación, para "expresar los variadísimos fenómenos que se originan en Cuba por las complejísimas transmutaciones de culturas que aquí se verifican" en todos los aspectos de la vida social e individual (1963: 99). La argumentación de Ortiz va en contra del uso del término aculturación, ya que éste no es suficiente para describir lo que resultó de esa curiosa mezcla de culturas, todas exógenas y desarraigadas de sus tierras originales, que fueron transplantadas a un mundo nuevo, donde predominaba la transitoriedad y todo estaba en constante recreación: “Hombres, economías, culturas y anhelos, todo aquí se sintió foráneo, provisional, cambiadizo, 'aves de paso' sobre el país, a su costa, a su contra y a su malgrado” (ibid.: 102).

Por ello, Ortiz considera que:

"el vocablo transculturación expresa mejor las diferentes fases del proceso transitivo de una cultura a otra, porque éste no consiste solamente en adquirir una cultura, que es lo que en rigor indica la voz anglo-americana 'aculturación', sino que el proceso implica también necesariamente la pérdida o desarraigo de una cultura precedente, lo que pudiera decirse una parcial desaculturación, y además, significa la consiguiente creación de nuevos fenómenos culturales que pudieran denominarse neoculturación" (ibid.: 103). 
La palabra transculturación ganó aceptación como categoría de análisis, seguramente debido a la potencia cognitiva que ofrece, y no solo en el campo de las ciencias sociales ya que Ángel Rama (1987) lo utiliza para sus estudios literarios y Nicholas Mirzoeff (2003) en el campo de los medios audiovisuales.

De acuerdo con Rama, la antropología latinoamericana rechazó el término 'aculturación' por las inferencias ideológicas que implicaba, dada su procedencia de la antropología anglosajona. Considera que de fondo Ortiz manifestó un rechazo a ver la cultura propia latinoamericana como una entidad pasiva, inferior, destinada a sufrir la mayor pérdida, incapaz de dar una respuesta creativa en el contacto. La valoración de Rama es que Ortiz logró redefinir el concepto de cultura como una energía creadora que actúa sobre la herencia particular y las aportaciones de la cultura con la que entra en contacto para elaborar con originalidad un producto nuevo, incluso a pesar de ubicarse en una circunstancia histórica adversa.

Lo que ocurrió con América a manos de los europeos sería un buen ejemplo para Mirzoeff de lo que llama el poder creador y destructor de la transculturación. Este autor considera que transcultura es el término idóneo para designar el nuevo tipo de cultura que se está gestando en nuestra época, de tipo visual, híbrida, producto de la mezcla de varias culturas, y que identifica lo dinámico y fluido del fenómeno contemporáneo. En referencia al papel que ahora ha adquirido la Otredad, destaca que la noción de transcultura posee un nuevo matiz, entendida como "la experiencia de la periferia sobre varios siglos pasados que ahora ha sido devuelta al remitente, ofreciendo un nuevo concepto de la propia cultura" (2003: 189). Es decir, la alteridad retorna fortalecida simbólicamente, como lo argumenta Rama y como lo implican todas las elaboraciones teóricas anteriores que nacen del pensamiento latinoamericano desde y para la población indígena.

\section{Un Síntesis Final: La Intertransculturalidad}

En el pensamiento del filósofo cubano Raúl Fornet-Betancour encontramos una formulación más elaborada y consistente de la relación entre la interculturalidad y la transculturación. Empieza 
por llevar estos términos a un nivel de discusión metacognitivo ya que problematiza el acto mismo de definir y, sobre todo, al hacerlo con la palabra interculturalidad, por las siguientes consideraciones:

1. Definir es un acto propio de la lógica de la cultura científica de Occidente.

2. Definir implica delimitar, fragmentar y parcelar, de acuerdo a los marcos de especialización científica, con la consecuencia de dificultar una posterior integración de las varias dimensiones desde las cuales se puede definir lo intercultural, y se corre el riesgo de perder la "substancia" de la noción, por convertirla en objeto de estudio.

3. Definir opera con base en el proceso de objetivar, que distancia al sujeto de su objeto de estudio, con lo cual se pierde de nuevo otro rasgo esencial: lo intercultural como algo de lo que somos parte y no un fenómeno lejano o externo a nuestra esfera de vida.

4. Las definiciones son apenas una pieza de un sistema teórico mayor que, nuevamente, responde a un ejercicio cognoscitivo monocultural (2001: 157-158).

En el espíritu propositivo de la reflexión filosófica, Fornet-Betancour sugiere, primero, entender la definición no como un acto que incluye totalmente lo que define, sino que un "simple punto de apoyo para comenzar el diálogo e intercambio con otros nombres posibles de aquello a lo que nos queremos aproximar con nuestra definición propia" (ibid.: 159). Esto implica colocarnos en un plano de analfabetismo cultural, como una postura abierta al aprendizaje de los distintos alfabetos de la diversidad (lingüística, cultural, étnica, religiosa, etc.). Así, mediante el proceso de alfabetización en la escuela del diálogo intercultural, se podrá ser conscientes de que 'nuestros' nombres son contextuales y que "necesitan ser redimensionados desde las perspectivas que se abren en los nombres de otras tradiciones culturales" (ibid.). Es decir, contrastar nuestras definiciones implica una voluntad explícita de la corrección mutua. De esta manera, el acto de definir se convierte en un acto verdaderamente intercultural que transforma las definiciones que sirven de punto de partida. Consideramos que esta voluntad y propuesta es coherente con la teoría de la acción comunicativa de 
Habermas, puesto que constituye un claro ejercicio de racionalidad dialógica e interacción comunicativa.

Fornet-Betancour procede a contrastar una serie de definiciones de la interculturalidad, como forma de debate, para demostrar que en este tipo de ejercicio se da un proceso de traducción de lo propio y de lo ajeno: "traducir al otro nuestra medida de las cosas y en el que aprendemos al mismo tiempo las (nuevas) dimensiones de las cosas en la visión de los otros" (ibid.: 160). Su valoración es que éste podría ser el sentido genuino de la interculturalidad, esa voluntad de traducción recíproca.

En un esfuerzo más complejo, el filósofo cubano propone más bien el neologismo intertransculturalidad. De acuerdo con Fajardo (2007), el prefijo 'inter' alude a la relación dialógica entre dos o más interlocutores que debe caracterizarse por actitudes de gran flexibilidad y apertura para asegurar su efectividad en los encuentros o confrontaciones con la diversidad: superar el etnocentrismo, enmendar las asimetrías de poder, disponibilidad para el cambio ("desobediencia cultural" en términos de Fornet-Betancour).

El siguiente prefijo, 'trans', indica la posibilidad de crear una razonabilidad cultural desde la cual ejercer la autocrítica y procurar el cambio interno "en el sentido de crecer y madurar en la humanitas” (2007: 2) y, además, 'dar a' y 'recibir de' el otro críticas a un nivel externo, todo ello como una dinámica de ayuda mutua hacia los valores humanos: "El trans- viene a ser la instancia mediadora que cualifica al inter- para avanzar en universalidad cultural, donde la diversidad sea admitida y celebrada como enriquecimiento mutuo" (ibid.).

De acuerdo con Fornet-Betancour, esta naturaleza de la cultura en permanente acción de modelar y ser modelada por la acción comunicativa en el espacio intersubjetivo de las relaciones sociales, la convierte en el sitio propio del ejercicio de la libertad humana, es decir, el punto de apoyo o espacio de mediación para la construcción del proyecto de vida ya que da "sustentabilidad a proyectos transformadores de largo alcance", para "el desafío vital pleno, gozando desde las herencias culturales propias y ajenas, de la 
factibilidad abierta para futuros diversos" (ibid.: 5).

En síntesis, la intertransculturalidad remite al tipo de convivencia en que, al acto de compartir (el momento inter-), le sigue el de dejarse transformar (el momento trans-), porque los modos de sentir se conjugan y crean un lenguaje común desde donde se puede ser autocríticos de la propia cultura, y de las otras visiones del mundo. $Y$ es por estas razones que la consideramos como una propuesta idónea para fundamentar el diseño de políticas educativas que se dirijan a legitimar y potenciar la educación intercultural bilingüe, puesto que guarda coherencia con las metas de aprendizaje que este modelo curricular persigue.

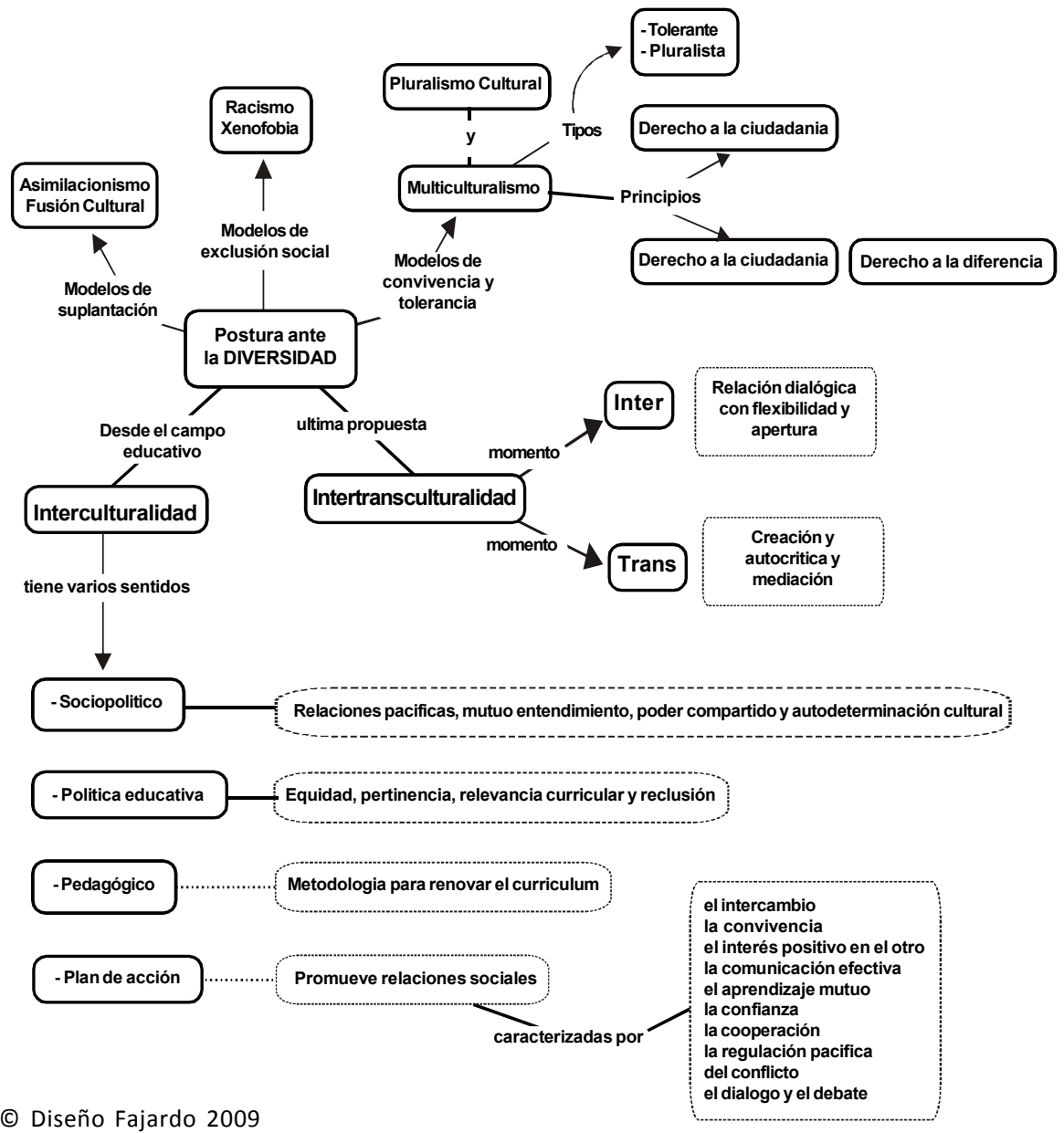




\section{Posturas ante la diversidad}

Extraído de: Fajardo, Delia (2009). La Educación Intercultural Bilingüe (EIB) en Honduras: teoría y didáctica para una adquisición de la competencia intercultural. Tesis de Doctorado para la obtención del título de Doctora en Enseñanza de la Lengua y la Literatura. Facultad de Formación del Profesorado. Universitat de Barcelona, Catalunya, España. 


\section{Bibliografía}

- Briones, G. (1999). La Teoría de la Acción Comunicativa de Jünger Habermas. Domen. Recuperado de http:// us.geocities.com/tomaustin_cl/soc/Habermas/haberesum.htm

- Díaz-Couder, E. (1998). Diversidad Cultural y Educación en Iberoamérica. Revista Iberoamericana de Educación 17: 11-30.

- Duarte Méndez, S. C. (coord.) (1999. Guía Sobre Interculturalidad. Primera Parte. Fundamentos Conceptuales. Guatemala: Proyecto Q'Anil / PNUD.

- Fajardo, J. M. (2007). La intertransculturalidad en el pensamiento de Raúl Fornet-Betancour. Potencialidades ÉticoPolíticas. Comunicación personal del autor vía correo electrónico. 7 de septiembre de 2007.

- Fornet-Betancour, R. (2001). Lo intercultural: el problema de su definición. Aulaintecultural. Recuperado de http:// www.aulaintercultural.org/IMG/pdf/betancour.pdf

- Malgesini, G. y Giménez, C. (2000). Guía de Conceptos sobre Migraciones, Racismo e Interculturalidad. Madrid: Catarata.

- Moya, R. (2005). Formación de Maestros e Interculturalidad. En Nucinkis, N. y López, L. E. (comps.). Formación Docente y Educación Intercultural Bilingüe en América Latina (CD-ROM). Bolivia: Ministerio de Educación de Bolivia / Cooperación Técnica Alemana (GTZ).

- Mirzoeff, N. (2003). Una Introducción a la Cultura Visual. Barcelona: Paidós.

- Perotti, A. (1994). The Case for intercultural education. Strasbourg: Council of Europe Press. 
- Ortiz, F. (1963). Contrapunteo Cubano del Tabaco y el Azúcar. La Habana: Consejo Nacional de Cultura.

- Rama, Á. (1982). Transculturación Narrativa en América Latina. México/Madrid: Siglo XXI, $1987^{3}$ 